\title{
A UNITARY AS A PRODUCT OF SYMMETRIES
}

\author{
N. K. THAKARE AND A. R. BALIGA \\ (Communicated by Ken Goodearl)
}

\begin{abstract}
It was proved by Fillmore that a unitary of a properly infinite von Neumann algebra $A$ can be expressed as a product of at most four symmetries. In this paper we introduce an axiom (ENCP) for Baer *-rings and prove that Fillmore's result is true if $A$ is a properly infinite Baer *-ring satisfying (ENCP) and $L P \sim R P$. This also affirmatively answers the open problem on $A W^{*}$ algebras posed by Berberian.
\end{abstract}

\section{INTRODUCTION}

In the operator algebra $B(\mathscr{H})$, there is a nice result that a unitary operator on an infinite-dimensional Hilbert space $\mathscr{H}$ is a product of at most four symmetries (selfadjoint unitaries). This was proved by Halmos and Kakutani [4]. This result was extended by Fillmore [3] to properly infinite von Neumann algebras. This prompted Berberian [2] to ask the following:

Open problem. Can a unitary of a properly infinite $A W^{*}$-algebra be expressed as a product of four symmetries?

The technique employed in [3] involves availability of the action of an element of a von Neumann algebra on a vector. Such actions of an element on a vector are not available for Baer *-rings. In this paper, using purely algebraic techniques, we show that for certain Baer *-rings, a unitary can be written as a product of at most four symmetries and as a consequence we affirmatively answer the above open problem.

All the terms that we have used in this paper are as per [2]. We shall not repeat the definitions of these terms. However, we may explain such concepts and notation that we mention but which do not appear in [2].

\section{NEW CONCEPTS AND PRELIMINARY RESULTS}

We begin with the definition of a new concept:

Received by the editors March 12, 1993 and, in revised form, June 25, 1993.

1991 Mathematics Subject Classification. Primary 16A28; Secondary 46K99, 46L99, 06A10.

Key words and phrases. Baer *-rings, $\mathrm{AW}^{*}$-algebras, unitary, symmetry in *-rings, existence of noncentral projection axiom, generalized comparability, $L P \sim R P$.

The second author gratefully acknowledges the financial assistance in the form of a Junior Research Fellowship from the U.G.C., New Delhi. 
2.1 Definition. A *-ring $A$ is said to satisfy the existence of noncentral projection axiom (briefly, the (ENCP)-axiom) if for every element $x \in A$, there exists a noncentral projection $p$, which commutes with $x$.

2.2 Remark. Clearly any abelian *-ring will not satisfy the (ENCP)-axiom.

The question that arises is whether every nonabelian $A W^{*}$-algebra satisfies the (ENCP)-axiom. We see that this is true.

\subsection{Proposition. Every nonabelian $A W^{*}$-algebra $A$ satisfies the (ENCP)- axiom.}

Proof. Let $x$ be a normal element in $A$. Then $x$ belongs to some maximal abelian selfadjoint subalgebra $M[2$, p. 9]. If there exists a noncentral projection, $p$, in $M$, then $p x=x p$ since $M$ is abelian.

If every projection in $M$ is central, then $x$ belongs to the center since $M$ is the closed linear span of its projections [2, p. 43]. Since $A$ is nonabelian, there exists a noncentral projection $p$ in $A$, and hence $p x=x p$.

2.4 Lemma. Let $A$ be a nonabelian Baer *-ring with Generalized Comparability $(G C)$ and satisfying (ENCP), $x$ be a normal element in $A$. There exists $a$ nonzero projection $p \in A$ such that

$$
p \precsim 1-p \text { and } p x=x p \text {. }
$$

Proof. This can be proved by the same way as the lemma in [3, p. 897] by using (ENCP) and (GC).

2.5 Remark. The above lemma could be proved using the weaker axiom of "Weak Generalized Comparability" (WGC) [1]. A ring is said to satisfy the Weak Generalized Comparability axiom (briefly, A satisfies (WGC)) if for every projection $e$, there exists a central projection $h$ such that

$$
h e \precsim h(1-e) \text { and }(1-h)(1-e) \precsim(1-h) e \text {. }
$$

But in the theorems that follow, (WGC) cannot be used.

The next remark is required several times.

2.6 Remark. If $p$ is a properly infinite projection in a Baer *-ring $A$ with the parallelogram law $(\mathrm{P})$ and if $p_{1}, p_{2}, \ldots$ are projections in $A$ with $p_{n} \precsim p$ for all $n$, then $\sup p_{n} \precsim p$.

Indeed, using the fact that (P) implies (GC) for Baer *-rings [7, Theorem 2.1], the above remark can be proved in the same way as the remark in [3], by using $(P)$ and [2, Theorem 1, p. 103].

2.7 Lemma. Let $A$ be a Baer *-ring with (GC) and with no abelian direct summand. Let $\left(e_{i}\right)_{i \in I}$ and $\left(f_{i}\right)_{i \in I}$ be orthogonal families of projections such that $e_{i} \sim f_{i}$ via the partial isometry $w_{i}$ for every $i$ and $\sup e_{i}=\sup f_{i}=1$. Then there exists a unitary $v$ such that $v e_{i}=f_{i} v=w_{i}$ and $v v^{*}=v^{*} v=1$.

Proof. The partial isometries $w_{i}$ are addable by Theorem 1 in [2, p. 129]. Hence, there exists $v$ such that

$$
v e_{i}=f_{i} v=w_{i} \text { and } v v^{*}=v^{*} v=1 .
$$




\section{A UNITARY AS A PRODUCT OF SYMMETRIES}

We now proceed to prove some main results which are the stepping stones to the stipulated result.

3.1 Theorem. Let $A$ be a finite Baer *-ring with (GC) and satisfying (ENCP). Let $x$ be a normal element in $A$ and $q$ be a projection in $A$. Then there exists a projection $p \in A$ such that $p \sim q$ and $p x=x p$.

Proof. This theorem can be proved in the same way as [3, Theorem 1] by using the following facts:

(1) There exists a unique dimension function $D$ for $A$ [2, p. 181].

(2) Every $x \in A$ has a central cover $C(x)[2$, p. 35].

(3) If $e, f$ are projections such that $C(e) \leq C(f)$ and if $e$ is abelian, then $e \precsim f[2, \mathrm{p} .112]$.

(4) If $p_{\alpha}$ is a family of projections with $p_{\alpha} \uparrow p$ and if $p_{\alpha} \precsim q$ for all $\alpha$, then $p \precsim q[2, \mathrm{p} .183]$.

(5) $p \sim p_{0}$ implies $1-p \sim 1-p_{0}[2$, p. 105].

3.2 Theorem. Let $A$ be a nonabelian Baer *-ring with $(L P \sim R P)$ and satisfying $(E N C P)$. Let $x$ be a normal element in $A$. Then there exist nonzero orthogonal, equivalent projections $p, q \in A$ each commuting with $x$.

Proof. Suppose first that there is in $A$ no nonzero finite projection which commutes with $x$. Then every projection which commutes with $x$ is properly infinite.

By Lemma 2.4, there is a projection $p \in A$ such that $p x=x p$ and $0 \neq$ $p \sim p_{0} \leq(1-p)$. Let $W$ be the multiplicative subsemigroup of $A$ generated by $x$ and $x^{*}$. Since $x$ is normal, $w \in W$ is of the form $x^{k}\left(x^{*}\right)^{s}$ for $k, s=$ $0,1,2, \ldots$. Hence, $1 \in W$. For $w \in W$, let $q_{w}=L P\left(w p_{0}\right)$ and let $q=$ $\sup \left(q_{w}: w \in W\right)$. Now $L P\left(w p_{0}\right) \sim R P\left(w p_{0}\right) \leq p_{0}$. Note that $(L P \sim R P)$ implies (P) [2, p. 63], and hence it implies (GC) (Remark 2.6). Using the above facts, Lemma 2.4, Remark 2.6, and Theorem 3.1 we can complete the proof of this theorem in much the same manner as that of [3, Theorem 2].

3.3 Theorem. Let $A$ be a properly infinite Baer *-ring with $(L P \sim R P)$ and satisfying $(E N C P)$. Let $x$ be a normal element in $A$. Then there exists a sequence $\left\{p_{n}\right\}_{n=1}^{\infty}$ of orthogonal, equivalent projections in $A$, each commuting with $x$, whose sum is 1 .

Proof. Using Theorem 3.2, this theorem can be proved in the same way as [3, Theorem 3].

3.4 Theorem. If $A$ is a properly infinite Baer *-ring with $(L P \sim R P)$ and satisfying $(E N C P)$, then a unitary of $A$ is a product of at most four symmetries from $A$.

Proof. By Theorem 3.3, there exists a sequence $\left\{p_{n}:-\infty<n<\infty\right\}$ of orthogonal, equivalent projections each commuting with the unitary $u$ and with sum 1. For each $n$, select $x_{n} \in A$ with $x_{n}^{*} x_{n}=p_{n}$ and $x_{n} x_{n}^{*}=p_{n+1}$. By Lemma 2.7 there exists a unitary $v$ such that $v p_{n}=p_{n+1} v=x_{n}$. Let $w=v^{*} u$. Then $v$ and $w$ are unitaries. We next show that each is a product of two symmetries. We have $v^{k} p_{n} v^{-k}=p_{n+k}$ for each integer $k$, since $v p_{n} v^{-1}=p_{n+1}$ and $v^{-1} p_{n} v=p_{n-1}$. We put $w_{n}=v^{1-2 n} p_{n}$. Then $w_{n}^{*} w_{n}=p_{n}$ and $w_{n} w_{n}^{*}=p_{1-n}$. 
By Lemma 2.7 again there exists a unitary $s$ such that $s p_{n}=p_{1-n} s=w_{n}$. Moreover, we have $s=s^{*}$, since

$$
p_{n} s=w_{1-n}=v^{2 n-1} p_{1-n}=\left(v^{2 n-1} p_{1-n} v^{1-2 n}\right) v^{2 n-1}=p_{n} v^{2 n-1}=w_{n}^{*}=p_{n} s^{*}
$$

for every $n$ and $\sup p_{n}=1$. Hence, $s$ is a symmetry.

Next, we put $u_{n}=v^{-2 n} p_{n}$. Then $u_{n}^{*} u_{n}=p_{n}$ and $u_{n} u_{n}^{*}=p_{-n}$. By the same way as above, there exists a symmetry $t$ such that $t p_{n}=p_{-n} t=u_{n}$. Then we have $s t=v$, since

$$
s t p_{n}=s u_{n}=s v^{-2 n} p_{n}=s p_{-n} v^{-2 n}=w_{-n} v^{-2 n}=v^{1+2 n} p_{-n} v^{-2 n}=v p_{n} .
$$

Similarly we can express $w$ as a product of two symmetries, for we have $p_{n} w=$ $p_{n} v^{*} u=v^{*} p_{n+1} u=v^{*} u p_{n+1}=w p_{n+1}$.

Finally, since $u=v w, u$ is expressed as a product of four symmetries.

3.5 Corollary. A unitary of a properly infinite $A W^{*}$-algebra can be expressed as a product of four symmetries.

Proof. ( $L P \sim R P)$ holds in any AW*-algebra; see [5, p. 44]. Also by Proposition 2.3 any nonabelian $\mathrm{AW}^{*}$-algebra satisfies the (ENCP)-axiom. Hence the conditions of Theorem 3.4 are satisfied in a properly infinite $\mathrm{AW}^{*}$-algebra and the conclusion follows.

\section{ACKNOWLEDGMENT}

The authors are grateful to the referee for suggesting shorter proofs of almost all the results of the paper.

\section{REFERENCES}

1. E. P. Armendariz and S. K. Berberian, Baer rings satisfying $J^{2}=J$ for all ideals, Comm. Algebra 17 (1989), 1939-1958.

2. S. K. Berberian, Baer *-rings, Springer-Verlag, Berlin, 1972.

3. P. A. Fillmore, On products of symmetries, Canad. J. Math. 18 (1966), 897-900.

4. P. R. Halmos and S. Kakutani, Products of symmetries, Bull. Amer. Math. Soc. 64 (1958), 77-78.

5. I. Kaplansky, Projections in Banach algebras, Ann. of Math. (2) 53 (1951), 235-249.

6. I. Kaplansky, Rings of operators, Benjamin, New York, 1968.

7. S. Maeda and S. S. Holland, Jr., Equivalence of projections in Baer *-rings, J. Algebra 39 (1976), 150-159.

Department of Mathematics, North Maharashtra University, Jalgaon 425001, India

Department of Mathematics, University of Poona, Pune 411007, India

Current address, A. R. Baliga: RMIT, Melbourne, 3000 Victoria, Australia 